\title{
Filamentous, Helical, and Tubular Microstructures during Cholesterol Crystallization from Bile Evidence that Cholesterol Does Not Nucleate Classic Monohydrate Plates
}

\author{
Fred M. Konikoff, " Doo Soo Chung, ${ }^{\ddagger}$ Joanne M. Donovan, *' Donald M. Small," and Martin C. Carey * \\ *Department of Medicine, Brigham and Women's Hospital, Harvard Medical School and Harvard Digestive Diseases Center, \\ Boston, Massachusetts 02115; ${ }^{\ddagger}$ Department of Physics, and Center for Materials Science and Engineering, \\ Massachusetts Institute of Technology, Cambridge, Massachusetts 02139; $\$$ Department of Medicine, Brockton/West Roxbury \\ Veterans Administration Medical Center, West Roxbury, Massachusetts 02132; \\ and "Department of Biophysics, Boston University Medical Center, Boston, Massachusetts 02118
}

\begin{abstract}
Precipitation of cholesterol in gallbladder bile is believed to produce platelike cholesterol monohydrate crystals directly. We report complementary time-lapse microscopic studies of cholesterol crystallization from model bile that reveal initial assembly of filamentous cholesterol crystals covered by a monomolecular layer of lecithin. Over a few days, the filaments evolved through needle, helical, and tubular microstructures to form classical platelike cholesterol monohydrate crystals. Similar crystallization phenomena were observed in human gallbladder biles from cholesterol but not pigment stone patients. Synchrotron $x$-ray diffraction of the earliest filaments suggested a cholesterol monohydrate polymorph or admixture with an anhydrous cholesterol precursor. However, density gradient centrifugation of filamentous crystals revealed that their density was $1.032 \mathrm{~g} / \mathrm{ml}$, consistent with anhydrous cholesterol. Conventional $x$-ray diffraction of transitional crystalline forms was consistent with pure cholesterol monohydrate crystals, as were the equilibrium platelike crystals. These novel findings suggest that crystalline cholesterol in bile may not be completely mature or hydrated initially, but undergoes a series of transformations to become thermodynamically stable monohydrate plates. These observations have important implications for understanding the control of cholesterol crystallization in bile, as well as explaining putative crystal cytotoxicity during gallstone formation. ( J. Clin. Invest. 1992. 90:11551160.) Key words: nucleation $\bullet$ microscopy $\bullet$ synchrotron $x$-ray diffraction $\bullet$ crystal structure $\bullet$ gallstones
\end{abstract}

This paper was presented in part at the annual meeting of the American Association for the Study of Liver Diseases, Chicago, IL, 2-5 November 1991, and published in abstract form (1991. Hepatology. 14:135A).

Address correspondence to Martin C. Carey, M.D., GI Division, Department of Medicine, Brigham and Women's Hospital, 75 Francis St., Boston, MA 02115. Dr. F. Konikoff's present address is Dept. of Gastroenterology, Ichilov Hospital, Tel-Aviv Medical Center, 6 Weizman Street, Tel-Aviv, Israel.

Received for publication 20 February 1992 and in revised form 1 June 1992.

J. Clin. Invest.

(c) The American Society for Clinical Investigation, Inc.

$0021-9738 / 92 / 09 / 1155 / 06 \$ 2.00$

Volume 90, September 1992, 1155-1160

\section{Introduction}

Cholesterol is eliminated from blood and other tissues via lipoprotein endocytosis, hepatic catabolism to bile salts, and secretion with lecithins and bile salts into bile $(1,2)$. Although virtually insoluble in water $\left(\sim 10^{-8} \mathrm{M}\right)(3), 5-50 \mathrm{mM}$ cholesterol is solubilized in human gallbladder bile within thermodynamically stable bile salt/lecithin micelles, and frequently metastable unilamellar lecithin vesicles $(2,4)$. Nucleated bile is typified by platelike cholesterol monohydrate crystals (5) that agglomerate via mucin glycoproteins to form cholesterol gallstones (6) found in at least $10 \%$ of Western humans (7). Cholesterol crystallization is believed to follow aggregation (8) and possibly fusion (9) of cholesterol-rich vesicles, but the crystallization process is poorly understood. In this study we present polarizing, phase-contrast, video-enhanced, fluorescence and electron microscopy, as well as x-ray diffraction and density data of early cholesterol crystallization phenomena in bile. Our studies reveal novel cholesterol crystalline habits, x-ray diffraction patterns, and crystal densities that challenge currently held beliefs that cholesterol crystallizes in bile directly as thermodynamically stable platelike monohydrate crystals.

\section{Methods}

\section{Chemicals}

Sodium salts of taurocholate (TC) ${ }^{1}$ and taurodeoxycholate (TDC) were obtained from Sigma Chemical Co. (St. Louis, MO). TC was recrystallized (10), and both TC and TDC were found to be $>98 \%$ pure by HPLC and TLC. Cholesterol (Nu Chek Prep Inc., Elysian, $\mathrm{MN}$ ) and grade I egg yolk lecithin (EYL) (Lipid Products, South Nutfield, Surrey, U.K.) were found to be $>99 \%$ pure by TLC, GC, and HPLC. $N$-(lissamine rhodamine B sulfonyl)-phosphatidylethanolamine (R-PE) was from Avanti Polar Lipids, Inc. (Birmingham, AL), and $\left[{ }^{3} \mathrm{H}\right]$ cholesterol and $\left[{ }^{14} \mathrm{C}\right] \mathrm{sn}-1-16: 0: \mathrm{sn}-2-18: 2$ lecithin were from New England Nuclear (Boston, MA). All other chemicals and solvents were American Chemical Society or reagent grade quality (Fisher Scientific Co., Pittsburgh, PA). For standards, cholesterol monohydrate and

1. Abbreviations used in this paper: CSI, cholesterol saturation index; EYL, egg yolk lecithin; GC, gas-liquid chromatography; QLS, quasielastic light scattering spectroscopy; $\overline{\mathrm{R}}_{\mathrm{h}}$, mean hydrodynamic radius; R-PE, $N$-(lissamine rhodamine B sulfonyl)-phosphatidylethanolamine; TC, taurocholate; TDC, taurodeoxycholate. 
anhydrous cholesterol crystals were grown from $95 \% \mathrm{EtOH}$ and $100 \%$ acetic acid respectively (11). $\mathrm{NaCl}$ was heated for $3 \mathrm{~h}$ at $600^{\circ} \mathrm{C}$ to oxidize and remove organic impurities. Pyrex brand glassware was alkali-alcohol washed (EtOH - $2 \mathrm{M} \mathrm{KOH} \mathrm{1:1,} \mathrm{vol/vol),} \mathrm{and} \mathrm{acid}$ washed ( $1 \mathrm{M} \mathrm{HNO}_{3}$ ), for $24 \mathrm{~h}$ each, and then rinsed thoroughly with purified water before oven drying. Water was filtered, ion exchanged, and glass distilled (Corning Glass Inc., Corning, NY).

\section{Model biles}

Cholesterol, EYL, and TC were coprecipitated from chloroform-methanol ( $2: 1 \mathrm{vol} / \mathrm{vol})$ and dried under $\mathrm{N}_{2}$ and reduced pressure. The dried

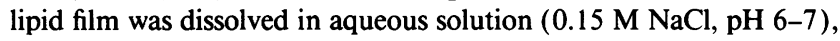
containing $3 \mathrm{mM} \mathrm{NaN}_{3}$ as an antimicrobial agent. Total lipid concentration was designed so that relative lipid composition plotted within the micellar phase limits of an appropriate phase diagram (12). To obviate possible persistence of undissolved microcrystalline cholesterol and to ensure complete micellization, the system was incubated for $1 \mathrm{~h}$ at $60^{\circ} \mathrm{C}$. To induce supersaturation, the stock solution was diluted with $0.15 \mathrm{M} \mathrm{NaCl}$ so that the relative lipid composition plotted above the contracted micellar phase limit (12). This dilution time-point was taken as the initiation of the nucleation process.

\section{Human biles}

Fresh gallbladder biles (generous gifts of Dr. M. Cahalane, Beth Israel Hospital, Boston, MA) were obtained from patients undergoing cholecystectomy after written informed consent and approval by the institutional human subjects committee. Samples were cultured to exclude infection, lipid analysis was performed (described below), and the cholesterol saturation index (CSI) was calculated (12). Gallstones were washed with distilled water and their cholesterol content ( $w t / w t)$ was measured by GC (Shimadzu GC-9A, Kyoto, Japan ) using stigmasterol as internal standard (13). Stones with a cholesterol content of $<20 \%$ or $>80 \%$ were classified as pigment or cholesterol stones, respectively. After stone removal, unprocessed biles were followed by sequential microscopic examinations. Throughout all experiments, biles (model and native) were kept at $37^{\circ} \mathrm{C}$ in sealed glass tubes, under argon.

\section{Analytical procedures}

Light microscopy. Bile samples ( $10 \mu \mathrm{l})$ were placed on a glass slide at room temperature and observed initially without a cover slip using a polarizing microscope (Photomicroscope III; Carl Zeiss, Inc., Thornwood, NY.) Then the sample was compressed with a cover glass and reexamined using phase contrast optics or a Zeiss Axiovert $35 \mathrm{M}$ microscope equipped with Nomarski differential interference contrast optics. For video-enhanced time-lapse microscopy, 200- $\mu$ l samples were incubated in a sealed drop-slide (Fisher Scientific Co.) on a heating stage (HS-1; Instec, Boulder, CO) at controlled (Apple Ile computer) temperature $\left(37.0 \pm 0.1^{\circ} \mathrm{C}\right)$ attached to a microscope (Optiphot-Pol; Nikon Inc., Instr. Group, Melville, NY) and sequentially photographed with a change-coupled device video camera (wv-GL1 10; Panasonic Industrial Co., Secaucus, NJ). Images were digitized from a video casette recording (AG-1960; Panasonic) by a frame grabber (Image Grabber; Neotech, Eastleigh, Hampshire, UK) and analyzed on a Macintosh IIci computer using Ultimage software (Graftek, Meudon-La-Foret, France).

Fluorescence microscopy. To enable morphologic localization of phospholipids during the crystallization process, model biles were prepared as described above, with traces of R-PE substituted for 0.5-5 $\mathrm{mol} \%$ of lecithin, as fluorescent probe. Samples were observed with a Zeiss Standard 16 fluorescence microscope equipped with phase contrast optics.

Electron microscopy. Samples for electron microscopy were obtained by aspirating agglomerated crystals via a wide bore needle, or after sedimentation of crystals by centrifugation. Scanning electron microscopy ( JSM-35CF; JEOL, Peabody, MA) was performed after air drying and gold coating, and negative stain transmission electron microscopy (Philips 300; Philips Electronic Instr. Co., Mahwah, NJ) was performed after staining with $1.5 \%$ phosphotungstic acid and drying in air.

Quasielastic light scattering spectroscopy (QLS). Measurements were performed at a constant scattering angle $\left(90^{\circ}\right)$ and controlled temperature $\left(37^{\circ} \mathrm{C}\right)$ on a home-built apparatus (14), equipped with a digital autocorrelator (BI2030AT; Brookhaven Instruments Corp., Holtsville, NY). Mean hydrodynamic radii $\left(\bar{R}_{h}\right)$ of particles in solution were derived from experimentally determined mean diffusion coefficients, and where appropriate, multicomponent analysis was used to confirm the presence of two or more discrete particle populations (14).

Gel permeation chromatography. To quantify micellar and vesicular fractions that solubilize cholesterol, bile samples were chromatographed on a high performance Superose six-gel filtration column as described previously (15) with $16 \mathrm{mM}$ TC as eluent. This TC concentration was determined to be the intermixed micellar/vesicular bile salt concentration (16). Cholesterol and lecithin contents of eluted fractions radiolabeled with $\left[{ }^{3} \mathrm{H}\right]$ cholesterol and $\left[{ }^{14} \mathrm{C}\right] s n-1-16: 0: s n-2-18: 2$ lecithin were determined by dual radiocounting.

$X$-ray diffraction. Bile samples were concentrated by centrifugation and sealed in 1-mm quartz capillary tubes. Conventional $\mathrm{x}$-ray diffraction was performed using a Ni-filtered $\mathrm{CuK} \alpha$ beam from rotating anode generator (Gx-6; Elliot Automation, Borehamwood, UK) (11). Synchrotron $\mathrm{x}$-ray diffraction was performed at Brookhaven National Laboratories (Upton, NY, beam line X-20C) using a wavelength of $1.36 \AA$ A selected by a silicon crystal monochromator. Samples were continuously rotated through the long axis of the capillary tubes, perpendicular to the beam. For conventional and synchrotron $x$-radiations, exposure times were $18-72 \mathrm{~h}$ and $5 \mathrm{~min}-3 \mathrm{~h}$, respectively, and the diffraction patterns were recorded with position sensitive linear detectors.

Differential scanning calorimetry (DSC). High resolution DSC was performed on a calorimeter (MC-2; MicroCal Inc., Amherst, MA) connected to a personal computer that allowed automated control, data acquisition, and analysis. Samples were washed and diluted with degassed water and introduced at room temperature into the calorimeter cell $(1.5 \mathrm{ml})$, and run against a similar volume of water in a reference cell. Measurements were recorded over a temperature range of $10^{\circ} \mathrm{C}-100^{\circ} \mathrm{C}$ at a heating rate of $90^{\circ} \mathrm{C} / \mathrm{h}$.

Density gradient centrifugation. Crystals in nucleated bile samples were concentrated by centrifugation and layered on top of a linear (2-20\%) sucrose gradient in 13.2-ml nitrocellulose tubes, and centrifuged in a rotor (SW41; Beckman Instruments, Inc., Fullerton, CA) at $201,000 \mathrm{~g}$ at $20^{\circ} \mathrm{C}$ for $8 \mathrm{~h}$ (17). Fractions were collected from top to bottom with an L-shaped pasteur pipette and examined by phase contrast and polarizing light microscopy as described above. Sucrose densities were verified using a temperature compensated refractometer.

Lipid analysis. TC was quantified by the $3 \alpha$-hydroxysteroid dehydrogenase method (18), lecithin by an inorganic phosphorus procedure (19), and cholesterol enzymatically (20).

\section{Results}

The principal model bile used in our study was composed of TC, EYL, and cholesterol in a molar ratio of 97.5:0.8:1.7. When dissolved to a concentration of $7 \mathrm{~g} / \mathrm{dl}$, this mixture formed a micellar solution with $\overline{\mathrm{R}}_{\mathrm{h}}=17 \pm 5 \AA$ (mean $\pm \mathrm{SD}, n$ $=4$ ) by QLS. Sixfold dilution of the micellar solution increased the CSI from 90 to $208 \%$, and produced unilamellar vesicles $\left(\overline{\mathbf{R}}_{\mathrm{h}}=212 \pm 25 \AA\right.$ ) with a molar cholesterol/lecithin ratio $\sim 2$. The mixture was designed to have this vesicular lipid ratio $(4,12)$, and its composition was experimentally verified by gel permeation chromatography and analysis. By QLS, polydispersity increased monotonically over the first several hours of observation, and multicomponent analysis revealed at least two large $\left(\overline{\mathrm{R}}_{\mathrm{h}}>200 \AA\right)$ particle populations that coexisted with $>99 \%$ micelles (wt/wt), as derived by gel permeation chromatography. After $\sim 10 \mathrm{~h}$ into the nucleation sequence, reproduc- 

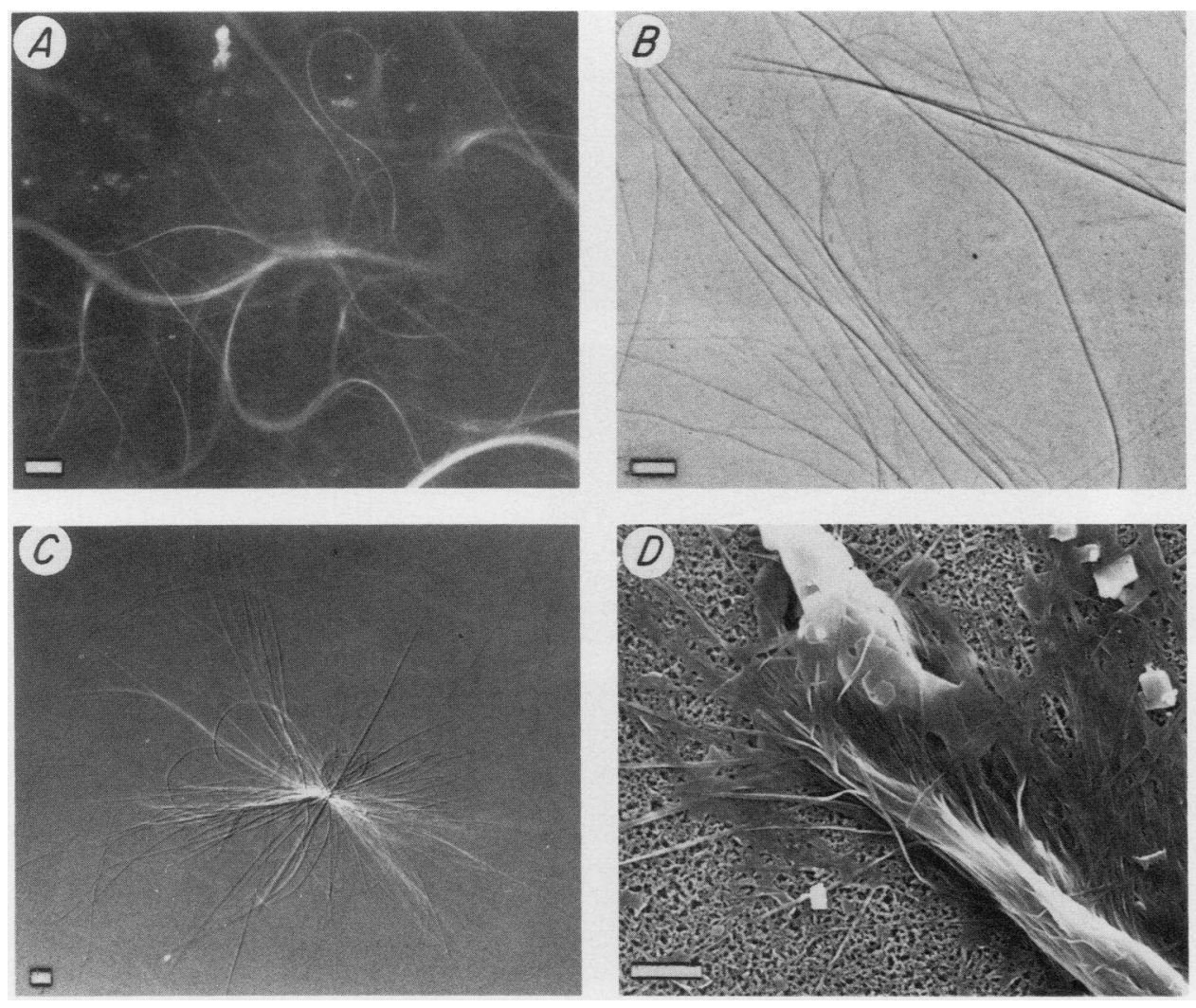

Figure 1. Photomicrographs of filamentous crystallization of cholesterol from model bile at $37^{\circ} \mathrm{C} .(A)$ Fluorescence microscopy (with $1 \%$ $\mathrm{R}-\mathrm{PE}$ ) at $4 \mathrm{~h}$ of nucleation. (B) Phase contrast microscopy at $6 \mathrm{~h}$. $(C)$ Light microscopy with Nomarski interference optics at $18 \mathrm{~h}$. (D) Scanning electron microscopy at $48 \mathrm{~h}$. Scale bars, $10 \mu \mathrm{m}$. ible autocorrelation functions could not be obtained because of large fluctuations in scattered light intensity.

Within 2-4 h of dilution, thin filamentous structures were observed by microscopy (Fig. 1). Individual filaments extended in length to several hundred micrometers (Fig. 1, $A$ and $B)$, and the width of the thinnest filaments was $0.1 \mu \mathrm{m}$ by scanning and transmission electron microscopy. Occasionally, filaments clumped together in "wheat-sheaf" bundles (Fig. 1, $C$ and $D$ ), and within 12-24 h, they had agglomerated into a buoyant wool-like macroscopic meshwork.

During 2-7 d, filaments were replaced by or coexisted with thicker needlelike and helical structures (Fig. 2) and platelike crystals. By time-lapse video-enhanced microscopy, filaments coiled into right-handed spirals and helices, which by lateral growth gradually formed crystalline tubelike structures (Fig. 2, $A-D)$. Eventually, the tubes fractured at their ends to produce platelike monohydrate crystals of typical habit (Fig. 2, $E$ and $F)$. Occasionally, filaments grew laterally to become flat ribbons that broke directly into plates (Fig. $1 D)$. As expected (4, 12), when crystals were separated centrifugally from the mother liquor at thermodynamic equilibrium (10-14 d), QLS analysis disclosed micelles $\left(\bar{R}_{h}=17 \pm 3 \AA\right)$, but no vesicles. Fig. 3 schematically depicts that the crystallization sequence could be divided into three stages, based on the principal crystalline habits present at each time period.

To obviate possible artifacts caused by undissolved microcrystals, incomplete hydration, or preparation path, the crystallization sequence was examined after preincubation $\left(20^{\circ} \mathrm{C}\right.$, $\left.37^{\circ} \mathrm{C}, 60^{\circ} \mathrm{C}, 1-24 \mathrm{~h}\right)$ or filtration $(0.22-\mu \mathrm{m}$ membranes; $\mathrm{Nu}-$ clepore, Corp., Pleasanton, CA ) of the micellar solution, and after preparing the nucleating bile by direct dilution of a dried lipid film to a final concentration of $1.2 \mathrm{~g} / \mathrm{dl}$. The crystalliza- tion process was found to be unaffected by all of these measures.

After ultracentrifugation $(210,000 \mathrm{~g}, 60 \mathrm{~min})$, filtration $(0.22 \mu \mathrm{m})$, and water-washing, chemical analysis of isolated filaments revealed a cholesterol/lecithin/bile salt molar ratio of 95.3:4.1:0.5. Assuming a close-packed lecithin monolayer (surface area of $65 \AA^{2}$ per molecule [21]), a filament of typical length $(100 \mu \mathrm{m})$ and diameter $(0.1 \mu \mathrm{m})$ would have a molar lecithin/cholesterol ratio of $3.8 \%$, which compares closely to the analytic value. After washing with $30 \mathrm{mM}$ TC and $50 \mathrm{mM}$ TDC, lecithin content was $1.7 \%$ and $0.9 \%$, respectively, suggesting that the monolayer was hydrophobically adsorbed. To verify this, fluorescence microscopy highlighted filaments grown from an identical model bile containing traces of R-PE (Fig. $1 A$ and Fig. 4). After addition of a few $\mu$ l of $50 \mathrm{mM}$ TDC, fluorescence was lost during $60 \mathrm{~min}$ without dissolution of the filaments (Fig. 4).

Fine structure of filaments at 24-48 h, when analyzed by conventional $\mathrm{x}$-ray powder diffraction, revealed three orders of diffraction maxima, corresponding to Bragg spacings of $34.1 \pm 1.4 \AA$, $17.2 \pm 0.3 \AA$, and $5.9 \pm 0.3 \AA$. This pattern was consistent with cholesterol monohydrate crystals $(11,22)$. In addition, scanning calorimetry disclosed an endothermic transition at $\sim 95^{\circ} \mathrm{C}$, and upon rescanning after rapid cooling a single endotherm at $\sim 36^{\circ} \mathrm{C}$, identical with the transitions obtained with a cholesterol monohydrate standard, and consistent with the known thermal behavior of cholesterol monohydrate (11). However, Fig. 5 shows that synchrotron $x$-ray diffraction of filaments harvested at 8-12 h, revealed in addition to the monohydrate pattern a peak at $4.9 \AA$, which corresponded precisely to a minor peak in the diffraction pattern of anhydrous cholesterol crystals. Density gradient centrifugation at $12 \mathrm{~h}$ resulted 

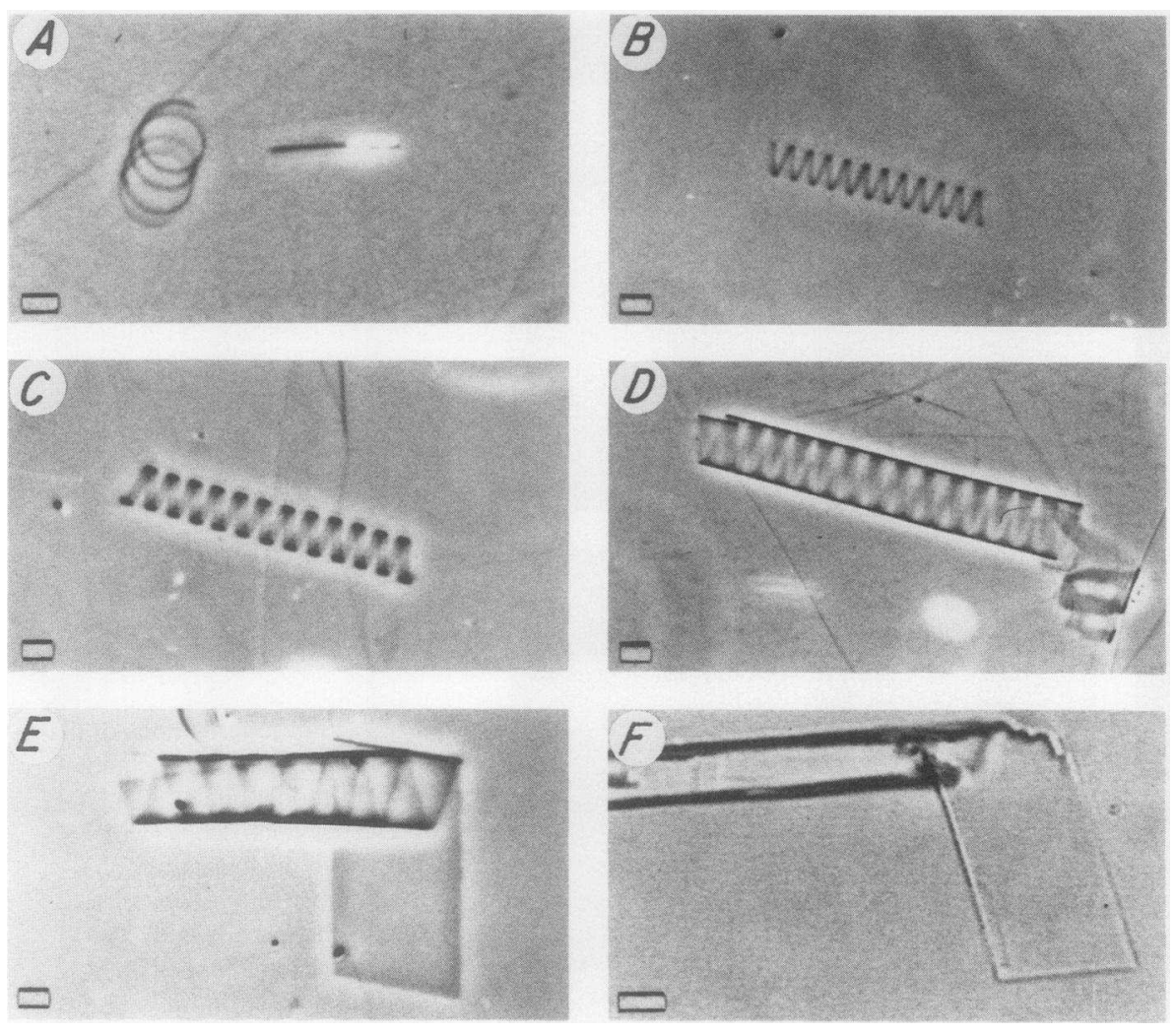

Figure 2. Representative time-lapse photomicrographs of crystalline cholesterol at days 2-6 by phase contrast $(A-E)$ and video-enhanced $(F)$ light microscopy. $(A)$ Filaments, a spiral, and a short needle on day 2. ( $B$ and $C$ ) Helices on days 3 and 4 . $(D$ and $E$ ) Microtubules with helical backbones producing platelike cholesterol crystals on day 5. $(F)$ A tube producing a platelike crystal of cholesterol monohydrate with a characteristic notched corner on day 6 . Scale bars, $10 \mu \mathrm{m}$. in a band of concentrated filamentous crystals at a density of $1.032 \mathrm{~g} / \mathrm{ml}$, compatible with anhydrous cholesterol (23), and a band consisting of platelike cholesterol monohydrate crystals at a density of $1.048 \mathrm{~g} / \mathrm{ml}$.

Supersaturated model biles simulating human gallbladder bile (TC/EYL/cholesterol 73.1:18.4:8.5; 6-10 g/dl, $n=8$ ) also revealed filamentous, helical, and tubular precipitates during the cholesterol crystallization process before the equilibrium state of platelike cholesterol monohydrate crystals was reached. However, compared to the dilute model system, filamentous crystals were less abundant in these biles and the time sequence of needle, helical, and tubelike growth was less predictable. Moreover, six of eight human gallbladder biles from cholesterol gallstone patients $(\mathrm{CSI}=92-273 \%)$, but not biles

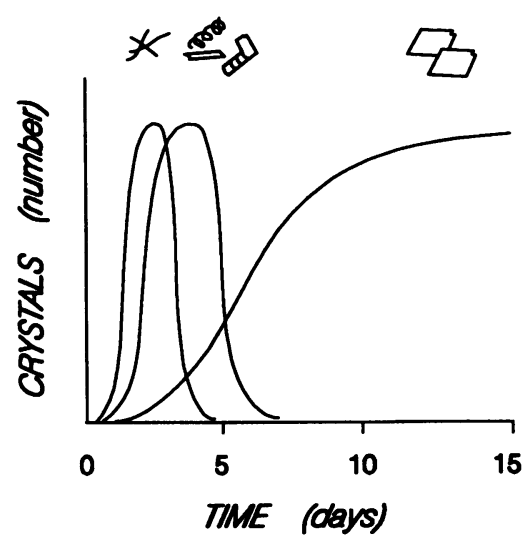

Figure 3. Schematic presentation of the three principal stages and crystal habits in the cholesterol crystallization sequence from model bile as functions of time. The initial stage is characterized by filaments, the transitional stage by helical and tubular structures, and the equilibrium stage by plates. The curves represent relative numbers of crystals (per high power microscopic field, all normalized to the same maximum). from four pigment gallstone patients $(\mathrm{CSI}=88-237 \%)$ also disclosed cholesterol filaments within 1-3 d of ex vivo incubation. Initially, six of the cholesterol stone biles, but no pigment stone biles, contained platelike cholesterol crystals, whereas by $30 \mathrm{~d}$ all cholesterol stone biles and one pigment stone bile showed platelike crystals. The amounts and time-sequences of nonplatelike crystals were variable and could not be correlated with chemical composition or CSI. In one cholesterol stone bile filamentous crystals were sufficiently abundant for isolation and analysis yielding a composition of $96.5 \%$ cholesterol and $3.5 \%$ lecithin.

\section{Discussion}

These experiments report an early crystallization sequence of cholesterol from supersaturated model and native biles in a series of crystal habits hitherto undocumented. Our results suggest that filamentous cholesterol crystals in bile express the habit and density of anhydrous cholesterol $(11,23)$. However, a needlelike crystal habit is not proof of an anhydrous state (24), and this habit may persist upon hydration (25). Moreover, although anhydrous and monohydrate cholesterol crystals are distinct and crystallographically well defined $(11,23$, 26), the predominant structural feature of early filaments in our study was that of cholesterol monohydrate with a minor reflection at $4.9 \AA$ corresponding to a reflection found in anhydrous cholesterol. This minor reflection could result from preferred orientation of longitudinal anhydrous crystals, or be part of the spectrum of a new crystalline cholesterol polymorph. It is extremely unlikely that the wide angle diffraction signal originates from the adsorbed lecithin monolayers, since the observed Bragg spacing of $4.9 \AA$ is too large, and the peak too sharp to arise from disordered lecithin chains $(21,27,28)$. 

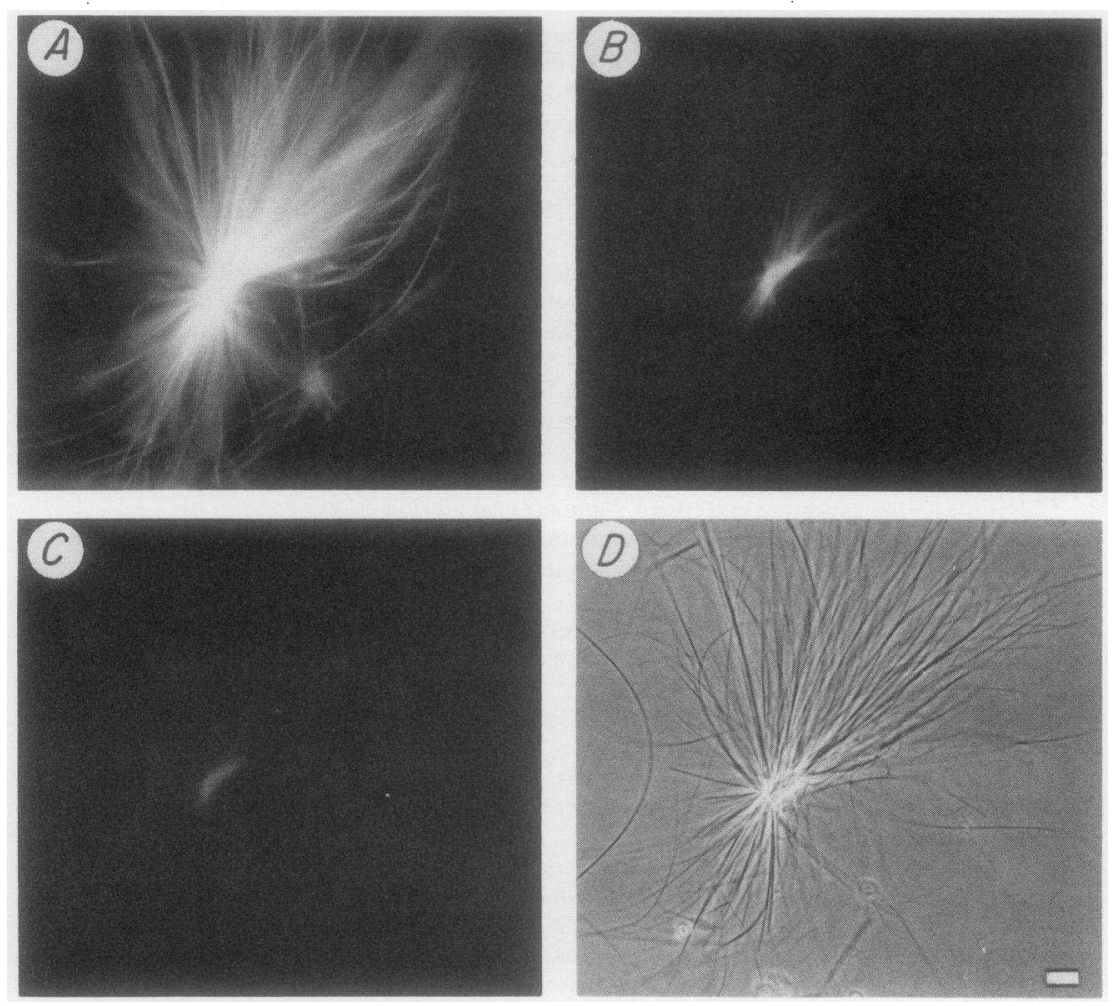

Figure 4. Photomicrographs of a "wheat-sheaf" bundle of cholesterol filaments grown from a bile containing $1 \mu \mathrm{M}$ R-PE (corresponding to $5 \mathrm{~mol} \%$ of lecithin content $)$. $(A-C)$ Fluorescence microscopy at $15 \mathrm{~min}, 30 \mathrm{~min}$, and $45 \mathrm{~min}$ after addition of $2 \mu \mathrm{l}$ of $50 \mathrm{mM}$ TDC under the microscope cover slip. This progressive loss of fluorescence was secondary to bile salt dissolution of surface lecithin molecules as verified analytically. $(D)$ Phase contrast microscopy of the same bundle of filaments at 60 min showing intact cholesterol crystals. Scale bar, $10 \mu \mathrm{m}$.
Hence, neither an intermediate cholesterol polymorph nor the terminal hydration of anhydrous cholesterol crystals can be ruled out with certainty, and definitive proof of the precise crystalline nature of the cholesterol filaments in bile must await additional studies.

The mechanisms of growth of filamentous cholesterol crystals and their transformations into helical and tubular microstructures are also unclear at present. Preferential unidimen-

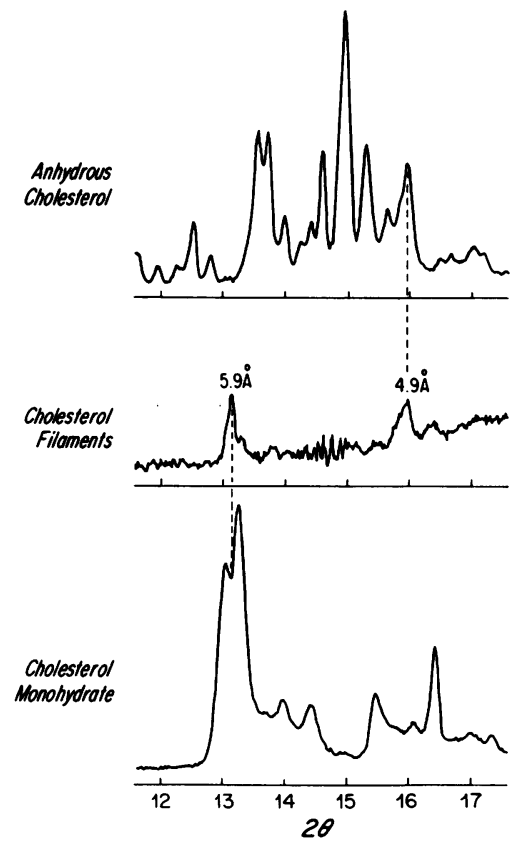

Figure 5. Synchrotron $\mathrm{x}$-ray diffraction patterns of cholesterol filaments and standards of cholesterol monohydrate and anhydrous crystals recorded by a position sensitive linear detector. Filaments were harvested at 8-12 $h$ of incubation and exposed for $30 \mathrm{~min}$ to the synchrotron $\mathrm{x}$-ray beam. The displayed portion of the diffraction spectrum of biliary cholesterol filaments $\left(2 \theta=11.3-17.8^{\circ}, \mathrm{d}\right.$ $=6.9-4.4 \AA$ ) highlights the $5.9 \AA$ reflection that overlaps with a major peak of cholesterol monohydrate, and the $4.9 \AA$ reflection that overlaps with a minor peak of anhydrous cholesterol crystals but has no counterpart in the spectrum of monohydrate cholesterol. sional growth could be influenced by chirality of the lecithin molecules in the surface monomolecular layer or the cholesterol molecules themselves, whether anhydrous or hydrated. Further, induced helical twists may result from preferential enantiomorphic packing of either surface (lecithin) or core (cholesterol) molecules. Helical and tubular habits have been observed in dilute crystalline suspensions of aqueous diacetylenic phospholipids and a number of other chiral amphiphiles with stiff (crystalline) chains (29-36). The needlelike habit is highly suggestive of anhydrous crystallization (11), which might be the case because in cholesterol-rich vesicles, the $3-\beta$ hydroxyl group of cholesterol and the $s n-2$ carbonyl of lecithin can be hydrogen bonded $(37,38)$. If initial crystallization is anhydrous, subsequent helical growth might be caused by asymmetric molecular strain during hydration as water molecules become incorporated into the crystals. It is also noteworthy that some typical platelike cholesterol crystals also appeared in the model system without going through any of the morphologic transitions described here. This is consistent with spectroscopic data (38), which suggest that as vesicles are depleted in cholesterol, the cholesterol molecules remaining within the lecithin bilayers may become hydrated.

Although alluded to previously (39), these novel crystal habits of cholesterol in bile have never been systematically evaluated as part of a cholesterol crystallization sequence $(8,40)$, nor have they been observed in other native systems, such as the developing atheromatous plaque (41). Even though the principal model system studied here is unphysiological in humans, the presence of filamentous cholesterol crystallization in both model biles of physiological composition and in native human gallbladder biles suggests that this phenomenon is physiologically relevant. Moreover, biliary lipid compositions similar to our model system have been reported in animals (42), and needlelike cholesterol crystals have been observed during 
cholesterol gallstone formation in the guinea pig (43). Nonetheless, it remains to be determined whether filamentous crystallization represents a major or a minor pathway of cholesterol crystallization in native biles. Further refinements of the physical-chemical properties of these crystallization phenomena are likely to lead to better insights on cholesterol nucleation and crystal growth in bile, and to identification of factors that may accelerate or retard these transitions. Moreover, it is intriguing to speculate that during gallbladder dehydration of bile, needlelike crystals may interact with apical membranes of mucosal cells, analogous to the "cytotoxic" effects of monosodium urate monohydrate and calcium pyrophosphate dihydrate crystals in the joints of gout and pseudo-gout patients (44).

\section{Acknowledgments}

We gratefully acknowledge the assistance of Dr. S. Hagen with electron and fluorescence microscopy and her critical review of the manuscript, Dr. S. Mochrie for his generosity with the synchrotron x-ray experiments, and Dr. A. Nusrat for Nomarski microscopy.

This work was supported by grants from the National Institutes of Health (DK 36588, DK 34854, and HL 26335, US Public Health Service) and the Veterans Administration; Dr. F. M. Konikoff was supported in part by a fund from The American Physicians Fellowship Inc.

\section{References}

1. Brown, M. S., and J. L. Goldstein. 1986. A receptor-mediated pathway for cholesterol homeostasis. Science (Wash. DC). 232:34-47.

2. Carey, M. C., and M. J. Cahalane. 1988. Enterohepatic circulation. In The Liver: Biology and Pathobiology. I. M. Arias, W. B. Jakoby, H. Popper, D. Schachter, and D. A. Shafritz, editors. Raven Press, Ltd., New York. 573-616.

3. Saad, H. Y., and W. I. Higuchi. 1965. Water solubility of cholesterol. $J$. Pharm. Sci. 54:1205-1206.

4. Cabral, D. J., and D. M. Small. 1989. Physical chemistry of bile. In Handbook of Physiology-The Gastrointestinal System III, Section 6. S. G. Schultz J. G. Forte, and B. B. Rauner, editors. American Physiological Society, Waverly Press, Baltimore, MD. 621-662.

5. Sedaghat, A., and S. M. Grundy. 1980. Cholesterol crystals and the formation of cholesterol gallstones. N. Engl. J. Med. 302:1274-1277.

6. Womack, N. A., R. Zeppa, and G. L. Irvin III. 1963. The anatomy of gallstones. Ann. Surg. 157:670-686.

7. Diehl, A. K. 1991. Epidemiology and natural history of gallstone disease. Gastroenterol. Clin. North Am. 20:1-19.

8. Holzbach, R. T. 1990. Nucleation of cholesterol crystals in native bile Hepatology. 12:155S-161S.

9. MacDonald, R. C. 1990. Surface chemistry of phospholipid vesicles relevant to their aggregation and fusion. Hepatology. 12:56S-61S.

10. Pope, J. L. 1967. Crystallization of sodium taurocholate. J. Lipid Res. 8:146-147.

11. Loomis, C. R., G. G. Shipley, and D. M. Small. 1979. The phase behavior of hydrated cholesterol. J. Lipid Res. 20:525-535.

12. Carey, M. C., and D. M. Small. 1978. The physical chemistry of cholesterol solubility in bile. Relationship to gallstone formation and dissolution in man. J. Clin. Invest. 61:998-1026.

13. Kern, F., Jr., H. Eriksson, T. Curstedt, and J. Sjövall. 1977. Effect of ethynylestradiol on biliary excretion of bile acids, phosphatidylcholines, and cholesterol in the bile fistula rat. J. Lipid Res. 18:623-634.

14. Cohen, D. E., M. R. Fisch, and M. C. Carey. 1990. Principles of laser light-scattering spectroscopy: applications to the physicochemical study of model and native biles. Hepatology. 12:113S-122S.

15. Cohen, D. E., and M. C. Carey. 1990. Rapid ( 1 hour) high performance gel filtration chromatography resolves coexisting simple micelles, mixed micelles, and vesicles in bile. J. Lipid Res. 31:2103-2112.

16. Donovan, J. M., N. Timofeyeva, and M. C. Carey. 1991. Influence of total lipid concentration, bile salt:lecithin ratio, and cholesterol content on inter- mixed micellar/vesicular (non-lecithin-associated) bile salt concentrations in model bile. J. Lipid Res. 32:1501-1512.

17. Renshaw, P. F., A. S. Janoff, and K. W. Miller. 1983. On the nature of dilute aqueous cholesterol suspensions. J. Lipid Res. 24:47-51.

18. Turley, S. D., and J. M. Dietschy. 1978. Re-evaluation of the $3 \alpha$-hydroxysteroid dehydrogenase assay for total bile acids in bile. J. Lipid Res. 19:924-928.

19. Bartlett, G. R. 1959. Phosphorous assay in column chromatography. $J$. Biol. Chem. 234:466-468.

20. Fromm, H., P. Amin, H. Klein, and I. Kupke. 1980. Use of a simple enzymatic assay for cholesterol analysis in human bile. J. Lipid Res. 21:259-261

21. Small, D. M. 1967. Phase equilibria and structure of dry and hydrated egg lecithin. J. Lipid Res. 8:551-557.

22. Katz, S. S., and D. M. Small. 1980. Isolation and partial characterization of the lipid phases of human atherosclerotic plaques. J. Biol. Chem. 255:97539759.

23. Shieh, H. S., L. G. Hoard, and C. E. Nordman. 1977. Crystal structure of anhydrous cholesterol. Nature (Lond.). 267:287-289.

24. Garti, N., L. Karpuj, and S. Sarig. 1981. Correlation between crystal habit and the composition of solvated and nonsolvated cholesterol crystals. J. Lipid Res. 22:785-791.

25. Stauffer, R. D., G. Bryson, and F. Bischoff. 1975. Cholesterol plate deposition in a pathological transport environment. Res. Commun. Chem. Pathol. Pharmacol. 11:515-522.

26. Craven, B. M. 1976. Crystal structure of cholesterol monohydrate. Nature (Lond.). 260:727-729.

27. Seul, M., P. Eisenberger, and H. M. McConnell. 1983. X-ray diffraction by phospholipid monolayers on single-crystal silicon substrates. Proc. Natl. Acad. Sci. USA. 80:5795-5797.

28. Janiak, M. J., C. R. Loomis, G. G. Shipley, and D. M. Small. 1974. The ternary phase diagram of lecithin, cholesteryl linoleate and water: Phase behavior and structure. J. Mol. Biol. 86:325-339.

29. Tachibana, T., and H. Kambara. 1969. Studies of helical aggregates of molecules. I. Enantiomorphism in the helical aggregates of optically active 12-hydroxystearic acid and its lithium salt. Bull. Chem. Soc. Jpn. 42:3422-3424.

30. Yamada, K., H. Ihara, T. Ide, T. Fukumoto, and C. Hirayama. 1984 Formation of helical super structure from single-walled bilayers by amphiphiles with oligo-L-glutamic acid-head group. Chem. Lett. 1713-1716.

31. Georger, J. H., A. Singh, R. R. Price, J. M. Schnur, P. Yager, and P. E. Schoen. 1987. Helical and tubular microstructures formed by polymerizable phosphatidylcholines. J. Am. Chem. Soc. 109:6169-6175.

32. Servuss, R. M. 1988. Helical ribbons of lecithin. Chem. Phys. Lipids. 46:37-41.

33. Nakashima, N., S. Asakuma, and T. Kunitake. 1985. Optical microscopic study of helical superstructures of chiral bilayer membranes. J. Am. Chem. Soc. 107:509-510.

34. Lin, K-C., R. M. Weis, and H. M. McConnell. 1982. Induction of helical liposomes by $\mathrm{Ca}^{2+}$-mediated intermembrane binding. Nature (Lond.). 296:164165.

35. Pfannemuller, B., and W. Welte. 1985. Amphiphilic properties of synthetic glycolipids based on amide linkages. I. Electron microscopic studies on aqueous gels. Chem. Phys. Lipids. 37:227-240.

36. Rudolph, A. S., B. R. Ratna, and B. Kahn. 1991. Self-assembling phospholipid filaments. Nature (Lond.). 352:52-55.

37. Wong, P. T. T., S. E. Capes, and H. H. Mantsch. 1989. Hydrogen bonding between anhydrous cholesterol and phosphatidylcholines: an infrared spectroscopic study. Biochim. Biophys. Acta. 980:37-41.

38. Sankaram, M. B., and T. E. Thompson. 1991. Cholesterol-induced fluidphase immiscibility in membranes. Proc. Natl. Acad. Sci. USA. 88:8686-8690.

39. LaMorte, W. W., B. F. Smith, and R. T. Holzbach. Discussants in: Lichtenberg, D., S. Ragimova, A. Bor, S. Almog, C. Vinkler, Y. Peled, and Z. Halpern. 1990. Stability of mixed micellar systems made by solubilizing phosphatidylcholine-cholesterol vesicles by bile salts. Hepatology. 12:149S-154S.

40. Toor, E. W., D. F. Evans, and E. L. Cussler. 1978. Cholesterol monohydrate growth in model bile solutions. Proc. Natl. Acad. Sci. USA. 75:6230-6234.

41. Small, D. M., and G. G. Shipley. 1974. Physical-chemical basis of lipid deposition in atherosclerosis. Science (Wash. DC). 185:222-229.

42. Khatim, M. S., K. A. Gumaa, O. Falk, and A. A. Osman. 1984. On the mechanism of decreasing the lithogenicity of bile in Camelus Dromedarius. Comp. Biochem. Physiol. 79A:379-382.

43. Jenkins, S. A. 1978. Biliary lipids, bile acids and gallstone formation in hypovitaminotic C guinea-pigs. Br. J. Nutr. 40:317-322.

44. Burt, H. M., J. K. Jackson, and W. Wu. 1991. Crystal-induced inflammation: studies of the mechanism of crystal-membrane interactions. Scanning Microsc. 5:273-280. 